\title{
The maximal theorem for weighted grand Lebesgue spaces
}

\author{
by \\ Alberto Fiorenza (Napoli), Babita Gupta (Delhi) \\ and PAnkaJ JAIN (New Delhi)
}

\begin{abstract}
We study the Hardy inequality and derive the maximal theorem of Hardy and Littlewood in the context of grand Lebesgue spaces, considered when the underlying measure space is the interval $(0,1) \subset \mathbb{R}$, and the maximal function is localized in $(0,1)$. Moreover, we prove that the inequality $\|M f\|_{p), w} \leq c\|f\|_{p), w}$ holds with some $c$ independent of $f$ iff $w$ belongs to the well known Muckenhoupt class $A_{p}$, and therefore iff $\|M f\|_{p, w} \leq c\|f\|_{p, w}$ for some $c$ independent of $f$.

Some results of similar type are discussed for the case of small Lebesgue spaces.
\end{abstract}

1. Introduction and preliminary results. The classical, celebrated Hardy inequality (see e.g. [8], [14], [16]) states that

TheOREM 1.1. Let $p>1$ and let $f$ be a measurable, nonnegative function in $(0,1)$. Then

$$
\left(\int_{0}^{1}(f f d t)^{p} d x\right)^{1 / p} \leq \frac{p}{p-1}\left(\int_{0}^{1} f^{p} d x\right)^{1 / p} .
$$

In this paper we first prove (see Theorem 2.1) the Hardy inequality in the context of the grand Lebesgue spaces $L^{p)}(0,1)$, introduced by Iwaniec and Sbordone in [10] (for properties and applications of these spaces see e.g. [7], [9], [2] and references therein), which are rearrangement-invariant Banach function spaces (see e.g. [1]) defined by the norm

$$
\|f\|_{p)}=\sup _{0<\varepsilon<p-1}\left(\varepsilon \int_{0}^{1}|f|^{p-\varepsilon} d t\right)^{\frac{1}{p-\varepsilon}}, \quad 1<p<\infty .
$$

We will state and use the properties of these spaces when they are needed; for brevity, we will write $L^{p)}$ instead of $L^{p)}(0,1)$ and in general, when the

2000 Mathematics Subject Classification: Primary 42B25; Secondary 46E30, 26 D15.

Key words and phrases: maximal function, Muckenhoupt weights, grand Lebesgue spaces, decreasing rearrangement, Hardy inequality. 
underlying measure space does not appear, we mean that it is the interval $(0,1)$ of the real line.

Here we just recall the continuous embeddings

$$
L^{p} \subset L^{p)} \subset L^{p-\varepsilon}, \quad 0<\varepsilon \leq p-1,
$$

and in the framework of Orlicz spaces, the continuous embeddings

$$
L^{p} \log ^{-1} L \subset L^{p)} \subset \bigcap_{\alpha<-1} L^{p} \log ^{\alpha} L .
$$

The inequality (1.1) is a tool for the proof of the boundedness of the Hardy-Littlewood maximal operator, defined by

$$
M f(x)=\sup _{(0,1) \supset I \ni x} \frac{1}{|I|} \int_{I}|f| d t, \quad x \in(0,1),
$$

where the supremum extends over all nondegenerate intervals, contained in $(0,1)$ and containing $x$, and $|I|$ denotes the Lebesgue measure of $I$. Such boundedness can be obtained (see e.g. [1, Thm. 3.10, p. 125]) through the notion of decreasing rearrangement of $f$, defined as

$$
f^{*}(t)=\sup _{|E|=t} \inf _{E} f, \quad t \in(0,1),
$$

where the supremum extends over all measurable sets $E \subset(0,1)$. An important relation between rearrangements and the maximal operator is given by the following well-known Herz theorem (see e.g. [1, Thm. 3.8, p. 122]), which establishes the equivalence of the functions $(M f)^{*}$ and the averaged rearrangement of $f$ (see [1, Def. 3.1, p. 52]) defined by

$$
f^{* *}(t)=\frac{1}{t} \int_{0}^{t} f^{*}(s) d s, \quad t \in(0,1) .
$$

THEOREM 1.2. There are absolute constants $c$ and $c^{\prime}$ such that for all $f \in L^{1}(0,1)$,

$$
c(M f)^{*}(t) \leq f^{* *}(t) \leq c^{\prime}(M f)^{*}(t), \quad t \in(0,1) .
$$

REMARK 1.3. The Herz theorem holds for functions defined on $\mathbb{R}^{n}$, $n \geq 1$, and in this case the constants $c$ and $c^{\prime}$ depend on the dimension $n$ only. A study of the constants $c$ and $c^{\prime}$ appears in Walker [20].

As a consequence of the Hardy inequality, we will show (see Corollary 2.3) that the classical maximal theorem of Hardy and Littlewood,

$$
\|M f\|_{p} \leq c\|f\|_{p},
$$

holds also when the norm $\|\cdot\|_{p}$ in the Lebesgue space $L^{p}$ is replaced by the corresponding one in the grand Lebesgue space $L^{p)}, 1<p<\infty$. In (1.2) and in the sequel, $c$ denotes a generic constant independent of the functions involved, and may change at each appearance. 
In Section 3, we introduce the weighted grand Lebesgue spaces $L_{w}^{p)}(0,1)$ with norm $\|\cdot\|_{p), w}$, and in Section 4 , we characterize the weights for which

$$
\|M f\|_{p), w} \leq c\|f\|_{p), w} .
$$

Namely, we prove that (1.3) holds iff $w$ belongs to the standard Muckenhoupt class $A_{p}$, defined in [15] (see also e.g. [19], [11], [4], [18], [12] for systematic treatments) through $(1<p<\infty)$

$$
\sup _{I}\left(\frac{1}{|I|} \int_{I} w d t\right)\left(\frac{1}{|I|} \int_{I} w^{-\frac{1}{p-1}} d t\right)^{p-1}=: A_{p}(w)<\infty,
$$

where the supremum extends over all intervals $I \subset(0,1)$. This class has been characterized as the class of weights such that

$$
\|M f\|_{p, w} \leq c\|f\|_{p, w}
$$

where $\|\cdot\|_{p, w}$ denotes the norm in the space $L_{w}^{p}$, given by

$$
\|f\|_{p, w}:=\left(\int_{0}^{1}|f|^{p} w d t\right)^{1 / p} .
$$

In other terms, we show that the class of weights which naturally could be named $A_{p}$ coincides in fact with $A_{p}$, i.e. the classes of weights for which the inequalities (1.4) and (1.3) are true are the same.

REMARK 1.4. The use of $A_{p}$ weights in connection with the Herz theorem appeared several years ago in a paper devoted to the study of the so-called reverse inequalities (see Sbordone [17]).

2. Hardy's inequality and maximal theorem for grand Lebesgue spaces (unweighted case). In this section we prove that the inequality (1.1) can be generalized to grand Lebesgue spaces.

Theorem 2.1. Let $1<p<\infty$. There exists a constant $c(p)>1$ such that

$$
\left\|\int_{0}^{x} f d t\right\|_{p)} \leq c(p)\|f\|_{p)}
$$

for all nonnegative measurable functions $f$ in $[0,1]$.

Proof. Let $0<\sigma<p-1$. We have

$$
\begin{aligned}
&\left\|\int_{0}^{x} f d t\right\|_{p)}=\max \left\{\sup _{0<\varepsilon<\sigma}\left(\varepsilon \int_{0}^{1}\left(f_{0}^{x} f d t\right)^{p-\varepsilon} d x\right)^{\frac{1}{p-\varepsilon}}\right. \\
&\left.\sup _{\sigma \leq \varepsilon<p-1}\left(\varepsilon \int_{0}^{1}\left(\int_{0}^{x} f d t\right)^{p-\varepsilon} d x\right)^{\frac{1}{p-\varepsilon}}\right\}
\end{aligned}
$$




$$
\begin{aligned}
& \leq \max \left\{\sup _{0<\varepsilon<\sigma}\left(\varepsilon \int_{0}^{1}\left(f_{0}^{x} f d t\right)^{p-\varepsilon} d x\right)^{\frac{1}{p-\varepsilon}},\right. \\
& \left.\quad\left(\sup _{\sigma \leq \varepsilon<p-1} \varepsilon^{\frac{1}{p-\varepsilon}}\right) \sigma^{-\frac{1}{p-\sigma}} \sigma^{\frac{1}{p-\sigma}}\left(\int_{0}^{1}\left(f_{0}^{x} f d t\right)^{p-\sigma} d x\right)^{\frac{1}{p-\sigma}}\right\} \\
& \leq(p-1) \sigma^{-\frac{1}{p-\sigma}} \sup _{0<\varepsilon \leq \sigma}\left(\varepsilon \int_{0}^{1}\left(\int_{0}^{x} f d t\right)^{p-\varepsilon} d x\right)^{\frac{1}{p-\varepsilon}} .
\end{aligned}
$$

Now take $0<\varepsilon \leq \sigma$, so that $p-\varepsilon>1$. Applying the Hardy inequality (1.1) with the exponent $p$ replaced by $p-\varepsilon$, and multiplying both sides by $\varepsilon^{\frac{1}{p-\varepsilon}}$, we get

$$
\left(\varepsilon \int_{0}^{1}(f f d t)^{p-\varepsilon} d x\right)^{\frac{1}{p-\varepsilon}} \leq \frac{p-\varepsilon}{p-\varepsilon-1}\left(\varepsilon \int_{0}^{1} f^{p-\varepsilon} d x\right)^{\frac{1}{p-\varepsilon}} .
$$

If we pass to the sup over $0<\varepsilon \leq \sigma$ on both sides, the previous inequality becomes

$$
\sup _{0<\varepsilon \leq \sigma}\left(\varepsilon \int_{0}^{1}\left(f_{0}^{x} f d t\right)^{p-\varepsilon} d x\right)^{\frac{1}{p-\varepsilon}} \leq \frac{p-\sigma}{p-\sigma-1} \sup _{0<\varepsilon \leq \sigma}\left(\varepsilon \int_{0}^{1} f^{p-\varepsilon} d x\right)^{\frac{1}{p-\varepsilon}}
$$

and therefore

$$
\left\|f_{0}^{x} f d t\right\|_{p)} \leq(p-1) \sigma^{-\frac{1}{p-\sigma}} \frac{p-\sigma}{p-\sigma-1} \sup _{0<\varepsilon<p-1}\left(\varepsilon \int_{0}^{1} f^{p-\varepsilon} d x\right)^{\frac{1}{p-\varepsilon}} .
$$

Setting

$$
c(p):=\inf _{0<\sigma<p-1}(p-1) \sigma^{-\frac{1}{p-\sigma}} \frac{p-\sigma}{p-\sigma-1}>1,
$$

we get the desired inequality (2.1).

REMARK 2.2. We wish to mention the important paper [13], where a condition on the upper Boyd index of a general rearrangement invariant space is given for the validity of the Hardy inequality. However, the proof of (2.1) would then be much less direct, and it would need the computation of the index (which is actually an open problem of independent interest).

We can now state the following corollary, whose proof is inspired by [1, Thm. 3.10, p. 125]. We remark that the general result by Lorentz and Shimogaki on the characterization of the rearrangement-invariant spaces on which the Hardy-Littlewood maximal operator is bounded (see e.g. [1, Thm. 5.17, p. 154]) cannot be applied, due to the fact that the underlying measure space is finite. 
Corollary 2.3. Let $1<p<\infty$. There exists a constant $c(p)>1$ such that

$$
\|M f\|_{p)} \leq c(p)\|f\|_{p)}
$$

for all $f \in L^{1}(0,1)$.

Proof. Since (see e.g. [1, Prop. 1.8, p. 43])

$$
\|f\|_{p}=\left\|f^{*}\right\|_{p}
$$

from Theorem 1.2 and from Theorem 2.1 applied to $f^{*}$ we get

$$
\|M f\|_{p)}=\left\|(M f)^{*}\right\|_{p)} \leq c\left\|f^{* *}\right\|_{p)} \leq c\left\|f^{*}\right\|_{p)}=c\|f\|_{p)},
$$

from which the assertion follows.

In the next section we will deal with the weighted case. We conclude this section by mentioning that together with the grand Lebesgue spaces the small Lebesgue spaces $L^{(p}(0,1)$ with norm $\|\cdot\|_{(p}$ are often considered, as associate spaces (see [1] for the definition of associate spaces, [5] for the definition of small Lebesgue spaces, and [2] and [3] for properties and references about small Lebesgue spaces). The Hardy inequality for small Lebesgue spaces is established in

Proposition 2.4. Let $1<p<\infty$. There exists a constant $C(p)>1$ such that

$$
\left\|\int_{0}^{x} f d t\right\|_{(p} \leq C(p)\|f\|_{(p}
$$

for all nonnegative measurable functions $f$ in $[0,1]$.

Proof. The inequality (2.3) can be easily deduced from the expression of the norm given in [6], using the rearrangement-invariance and the standard Hardy inequality:

$$
\begin{aligned}
\left\|\int_{0}^{x} f d t\right\|_{(p} & \leq c(p) \int_{0}^{1}(1-\log t)^{-1 / p}\left(\int_{0}^{t}\left(f_{0}^{x} f d s\right)^{p} d x\right)^{1 / p} d t / t \\
& \leq \frac{c(p) p}{p-1}\|f\|_{(p .}
\end{aligned}
$$

3. Weighted grand Lebesgue spaces. Let $w$ be a weight on $(0,1)$, i.e. an a.e. positive, integrable function on $(0,1)$, and $1<p<\infty$ be fixed. For all measurable, nonnegative, a.e. finite functions $f$ set

$$
\varrho(f):=\sup _{0<\varepsilon<p-1} \varepsilon^{\frac{1}{p-\varepsilon}}\|f\|_{p-\varepsilon, w}=\sup _{0<\varepsilon<p-1}\left(\varepsilon \int_{0}^{1} f^{p-\varepsilon} w d t\right)^{\frac{1}{p-\varepsilon}}
$$

It is straightforward to check the following: 
Proposition 3.1. The functional $\varrho$ is a (Banach) function norm, i.e. the following properties hold for all measurable, nonnegative, a.e. finite functions $f, g, g^{(n)}(n \in \mathbb{N})$, for all constants $\lambda \geq 0$, and for all measurable subsets $E \subset(0,1)$ :

- $\varrho(g) \geq 0$.

- $\varrho(g)=0$ iff $g=0$ a.e. in $(0,1)$.

- $\varrho(\lambda g)=\lambda \varrho(g)$.

- $\varrho(f+g) \leq \varrho(f)+\varrho(g)$.

- If $0 \leq g^{(n)} \uparrow g$ a.e. in $(0,1)$, then $\varrho\left(g^{(n)}\right) \uparrow \varrho(g)$.

- If $g \leq f$ a.e. in $(0,1)$, then $\varrho(g) \leq \varrho(f)$.

- $\varrho\left(\chi_{E}\right)<\infty$.

- $\int_{E} g w d x \leq C(p) \varrho(g)$ for some constant $C(p), 0<C(p)<\infty$, independent of $g$.

As a consequence, the space defined by the norm

$$
\|f\|_{p), w}:=\sup _{0<\varepsilon<p-1}\left(\varepsilon \int_{0}^{1}|f|^{p-\varepsilon} w d t\right)^{\frac{1}{p-\varepsilon}}
$$

is a Banach function space (see e.g. [1]), which we call the weighted grand Lebesgue space $L_{w}^{p)}(0,1)$. Except for the trivial case of $w$ constant, the space $L_{w}^{p)}(0,1)$ is not rearrangement-invariant.

REMARK 3.2. We observe that the extension of grand Lebesgue spaces to the weighted case has a relevant difference from the case of Lebesgue spaces. In the latter case, for a weight $w$, we have $f \in L_{w}^{p}$ (weighted Lebesgue space) if and only if $f w^{1 / p} \in L^{p}$ (unweighted Lebesgue space). This equivalence, however, is not true in grand Lebesgue spaces as can be seen from the following example.

ExAmPle 3.3. Let $\alpha>0$, and choose a weight $w(x)=x^{\alpha}$. Set $f(x)$ $=x^{\beta}, \beta>-\alpha-1$. It is straightforward to check that $f \in L_{w}^{p)}(0,1)$. On the other hand, for any choice of $\varepsilon, 0<\varepsilon<p-1$,

$$
\left(f w^{1 / p}\right)^{p-\varepsilon}=x^{(\beta+\alpha / p)(p-\varepsilon)},
$$

so that if we choose $\beta<-1-\alpha / p$, then the exponent on the RHS becomes smaller than -1 . Therefore $\left(f w^{1 / p}\right)^{p-\varepsilon}$ is not integrable in $(0,1)$ and so $f w^{1 / p} \notin L^{p)}(0,1)$.

The above example motivates us to define the space $\mathcal{L}_{w}^{p)}(0,1)$, related to the weighted grand Lebesgue space $L_{w}^{p)}(0,1)$, as

$$
\mathcal{L}_{w}^{p)}(0,1)=\left\{f:\left\||f| w^{1 / p}\right\|_{p)}<\infty\right\} .
$$


REMARK 3.4. It can be checked that $\mathcal{L}_{w}^{p)}(0,1)$ is also a Banach function space, and Example 3.3 shows that, in general, the spaces $L_{w}^{p)}(0,1)$ and $\mathcal{L}_{w}^{p)}(0,1)$ are different.

4. The main result. In this section we study the weighted version of (2.2), i.e.,

$$
\|M f\|_{p), w} \leq c\|f\|_{p), w} .
$$

In the framework of the standard Lebesgue spaces, it is well known that

$$
\|M f\|_{p, w} \leq c\|f\|_{p, w}
$$

if and only if $w$ satisfies the $A_{p}$ condition of Muckenhoupt ([15], [19]):

$$
\sup _{I \subset(0,1)} \frac{w(I)}{|I|}\left(\frac{1}{|I|} \int_{I} w^{-\frac{1}{p-1}}\right)^{p-1}<\infty,
$$

where $w(I)$ stands for $\int_{I} w d x$. The following result shows that condition (4.2) is necessary and sufficient for the validity of inequality (4.1) too.

Theorem 4.1. Let $1<p<\infty$ and $w$ be a weight on $(0,1)$. Then, in order that the inequality (4.1) be true with $c$ independent of integrable functions $f$ on $(0,1)$, it is necessary and sufficient that (4.2) holds, i.e., $w \in A_{p}$.

The proof of Theorem 4.1 uses the following well known lemma, proved in [15, Lemma 5, p. 214].

Lemma 4.2. If $1<p<\infty$ and $w \in A_{p}$ on $(0,1)$ with constant $A_{p}(w)$ $=K$, then there exist constants $\sigma>0$ and $L>0$ such that $w \in A_{p-\varepsilon}$ on $(0,1)$ with constant $A_{p-\varepsilon}(w) \leq L$, for all $0<\varepsilon<\sigma$.

Proof of Theorem 4.1. Let us first assume that the inequality (4.1) holds. We prove (4.2).

Fix an interval $I \subset(0,1)$. By the definition of maximal operator we have

$$
f_{I}|f| d x \leq M\left(f \chi_{I}\right)(x), \quad x \in I .
$$

On the other hand, by our assumption (4.1),

$$
\left\|M\left(f \chi_{I}\right)\right\|_{p), w} \leq c\left\|f \chi_{I}\right\|_{p), w} .
$$

By (4.3) and (4.4),

$$
\left(f_{I}|f| d x\right)\left\|\chi_{I}\right\|_{p), w}=\left\|f_{I}|f| d x \chi_{I}\right\|_{p), w} \leq\left\|M\left(f \chi_{I}\right)\right\|_{p), w} \leq c\left\|f \chi_{I}\right\|_{p), w}
$$




$$
\begin{aligned}
& =c \sup _{\varepsilon}\left(\varepsilon \int_{I} f^{p-\varepsilon} w d x\right)^{\frac{1}{p-\varepsilon}}=c \sup _{\varepsilon}\left(\varepsilon \int_{I} f^{p-\varepsilon} w^{\frac{p-\varepsilon}{p}} w^{\frac{\varepsilon}{p}} d x\right)^{\frac{1}{p-\varepsilon}} \\
& \leq c \sup _{\varepsilon} \varepsilon^{\frac{1}{p-\varepsilon}}\left(\int_{I}\left(f^{p-\varepsilon} w^{\frac{p-\varepsilon}{p}}\right)^{\frac{p}{p-\varepsilon}} d x\right)^{1 / p}\left(\int_{I}\left(w^{\frac{\varepsilon}{p}}\right)^{\frac{p}{\varepsilon}} d x\right)^{\frac{\varepsilon}{p(p-\varepsilon)}} \\
& =c \sup _{\varepsilon} \varepsilon^{\frac{1}{p-\varepsilon}}\left(\int_{I} f^{p} w d x\right)^{1 / p}\left(\int_{I} w d x\right)^{\frac{\varepsilon}{p(p-\varepsilon)}} \\
& =c\left(\int_{I} f^{p} w d x\right)^{1 / p} \sup _{\varepsilon} \varepsilon^{\frac{1}{p-\varepsilon}} w(I)^{\frac{\varepsilon}{p(p-\varepsilon)}} \\
& =c\left(\int_{I} f^{p} w d x\right)^{1 / p} \sup _{\varepsilon} \varepsilon^{\frac{1}{p-\varepsilon}} w(I)^{\frac{1}{p-\varepsilon}} w(I)^{-1 / p} \\
& =c w(I)^{-1 / p}\left(\int_{I} f^{p} w d x\right)^{1 / p} \sup _{\varepsilon} \varepsilon^{\frac{1}{p-\varepsilon}} w(I)^{\frac{1}{p-\varepsilon}} \\
& =c w(I)^{-1 / p}\left(\int_{I} f^{p} w d x\right)^{1 / p}\left\|\chi_{I}\right\|_{p), w}, \\
& =
\end{aligned}
$$

i.e.,

$$
f_{I}|f| d x \leq c w(I)^{-1 / p}\left(\int_{I} f^{p} w d x\right)^{1 / p} .
$$

Choosing now in the inequality above $f=w^{-\frac{1}{p-1}}$ we have $f^{p} w=w^{-\frac{1}{p-1}}$ and therefore

$$
\int_{I} w^{-\frac{1}{p-1}} d x \leq c w(I)^{-1 / p}\left(\int_{I} w^{-\frac{1}{p-1}} d x\right)^{1 / p}
$$

or

$$
w(I)^{1 / p}|I|^{-1}\left(\int_{I} w^{-\frac{1}{p-1}} d x\right)^{\frac{p-1}{p}} \leq c,
$$

from which, raising to the power $p$, we get (4.2).

To prove the converse, we begin by observing that by Lemma 4.2 there exists $0<\sigma<p-1$ such that

$$
\|M f\|_{p-\varepsilon, w} \leq c\|f\|_{p-\varepsilon, w}, \quad \varepsilon \in(0, \sigma],
$$

for some constant $c$ independent of $f$ and $\varepsilon$.

As a first step, consider $\varepsilon$ greater than $\sigma$, namely, fix $\varepsilon \in(\sigma, p-1)$, so that $(p-\sigma) /(p-\varepsilon)>1$. Applying the Hölder inequality with exponents $(p-\sigma) /(p-\varepsilon)$ and

$$
\left(\frac{p-\sigma}{p-\varepsilon}\right)^{\prime}=\frac{p-\sigma}{\varepsilon-\sigma}
$$


and taking into account that

$$
\left(1-\frac{p-\varepsilon}{p-\sigma}\right) \frac{p-\sigma}{\varepsilon-\sigma}=1
$$

we have

$$
\begin{aligned}
\|M f\|_{p-\varepsilon, w} & =\left[\int_{0}^{1}(M f)^{p-\varepsilon} w d x\right]^{\frac{1}{p-\varepsilon}} \\
& =\left[\int_{0}^{1}(M f)^{p-\varepsilon} w^{\frac{p-\varepsilon}{p-\sigma}} w^{1-\frac{p-\varepsilon}{p-\sigma}} d x\right]^{\frac{1}{p-\varepsilon}} \\
& \leq\left\{\int_{0}^{1}\left[(M f)^{p-\varepsilon} w^{\frac{p-\varepsilon}{p-\sigma}}\right]^{\frac{p-\sigma}{p-\varepsilon}} d x\right\}^{\frac{1}{p-\sigma}}\left[\int_{0}^{1}\left(w^{1-\frac{p-\varepsilon}{p-\sigma}}\right)^{\frac{p-\sigma}{\varepsilon-\sigma}} d x\right]^{\frac{\varepsilon-\sigma}{(p-\sigma)(p-\varepsilon)}} \\
& =\left[\int_{0}^{1}(M f)^{p-\sigma} w d x\right]^{\frac{1}{p-\sigma}}\left(\int_{0}^{1} w d x\right)^{\frac{\varepsilon-\sigma}{(p-\sigma)(p-\varepsilon)}} .
\end{aligned}
$$

Now, taking into account that

$$
\varepsilon \in(\sigma, p-1) \Rightarrow 0<\frac{\varepsilon-\sigma}{(p-\sigma)(p-\varepsilon)}<\frac{p-1-\sigma}{p-\sigma}
$$

and that

$$
\sigma<p-1 \Rightarrow(p-1) \sigma^{-\frac{1}{p-\sigma}}>1
$$

we have

$$
\begin{aligned}
& \|M f\|_{p), w}=\max \left\{\sup _{0<\varepsilon \leq \sigma} \varepsilon^{\frac{1}{p-\varepsilon}}\|M f\|_{p-\varepsilon, w}, \sup _{\sigma<\varepsilon<p-1} \varepsilon^{\frac{1}{p-\varepsilon}}\|M f\|_{p-\varepsilon, w}\right\} \\
& \leq \max \left\{\sup _{0<\varepsilon \leq \sigma} \varepsilon^{\frac{1}{p-\varepsilon}}\|M f\|_{p-\varepsilon, w},\right. \\
& \left.\leq \sup _{\sigma<\varepsilon<p-1} \varepsilon^{\frac{1}{p-\varepsilon}}\left[\int_{0}^{1}(M f)^{p-\sigma} w d x\right]^{\frac{1}{p-\sigma}}\left(\int_{0}^{1} w d x\right)^{\frac{\varepsilon-\sigma}{(p-\sigma)(p-\varepsilon)}}\right\} \\
& \leq \max \left\{1, \sup _{\sigma<\varepsilon<p-1} \varepsilon^{\frac{1}{p-\varepsilon}} \sigma^{-\frac{1}{p-\sigma}}\left(\int_{0}^{1} w d x\right)^{\frac{\varepsilon-\sigma}{(p-\sigma)(p-\varepsilon)}}\right\} \sup _{0<\varepsilon \leq \sigma} \varepsilon^{\frac{1}{p-\varepsilon}}\|M f\|_{p-\varepsilon, w} \\
& \leq c(p-1) \sigma^{-\frac{1}{p-\sigma}}\left(1+\int_{0}^{1} w d x\right)^{\frac{p-1-\sigma}{p-\sigma}}\|f\|_{p), w},
\end{aligned}
$$

and we are done. 
Acknowledgements. The first author has been supported by Italian M.I.U.R. research funds. The third author acknowledges with thanks the research grant no. SR/S4/MS:213/03 from the Department of Science and Technology (DST), New Delhi.

\section{References}

[1] C. Bennett and R. Sharpley, Interpolation of Operators, Academic Press, 1983.

[2] C. Capone and A. Fiorenza, On small Lebesgue spaces, J. Funct. Spaces Appl. 3 (2005), 73-89.

[3] G. Di Fratta and A. Fiorenza, A direct approach to the duality of grand and small Lebesgue spaces, Nonlinear Anal., to appear.

[4] J. Duoandikoetxea, Fourier Analysis, Grad. Stud. in Math. 29, Amer. Math. Soc., Providence, 2000.

[5] A. Fiorenza, Duality and reflexivity in grand Lebesgue spaces, Collect. Math. 51 (2000), 131-148.

[6] A. Fiorenza and G. E. Karadzhov, Grand and small Lebesgue spaces and their analogs, Z. Anal. Anwend. 23 (2004), 657-681.

[7] L. Greco, A remark on the equality $\operatorname{det} D f=\operatorname{Det} D f$, Differential Integral Equations 6 (1993), 1089-1100.

[8] G. H. Hardy, J. E. Littlewood and G. Pólya, Inequalities, Cambridge Univ. Press, 1952.

[9] T. Iwaniec, P. Koskela and J. Onninen, Mappings of finite distortion: Monotonicity and continuity, Invent. Math. 144 (2001), 507-531.

[10] T. Iwaniec and C. Sbordone, On the integrability of the Jacobian under minimal hypothesis, Arch. Ration. Mech. Anal. 119 (1992), 129-143.

[11] R. A. Kerman and A. Torchinsky, Integral inequalities with weights for the Hardy maximal function, Studia Math. 71 (1982), 277-284.

[12] V. Kokilashvili and M. Krbec, Weighted Inequalities in Lorentz and Orlicz Spaces, World Sci., River Edge, NJ, 1991.

[13] L. Maligranda, Generalized Hardy inequalities in rearrangement invariant spaces, J. Math. Pures Appl. 59 (1980), 405-415.

[14] V. G. Maz'ja, Sobolev Spaces, Springer, 1985.

[15] B. Muckenhoupt, Weighted norm inequalities for the Hardy maximal function, Trans. Amer. Math. Soc. 165 (1972), 207-226.

[16] B. Opic and A. Kufner, Hardy-Type Inequalities, Pitman Res. Notes Math. Ser. 219, Longman Sci. Tech., Harlow, 1990.

[17] C. Sbordone, Some reverse integral inequalities, Atti Accad. Pontaniana (N.S.) 33 (1984), 17-31.

[18] E. M. Stein, Harmonic Analysis: Real-Variable Methods, Orthogonality, and Oscillatory Integrals, Princeton Univ. Press, Princeton, 1993.

[19] A. Torchinsky, Real-Variable Methods in Harmonic Analysis, Academic Press, San Diego, 1986.

[20] P. L. Walker, Some estimates for the Hardy-Littlewood maximal function in $R^{n}$, Bull. London Math. Soc. 7 (1975), 139-143. 
Dipartimento di Costruzioni

e Metodi Matematici in Architettura

Università di Napoli

via Monteoliveto 3

80134 Napoli, Italy

E-mail: fiorenza@unina.it

and

Istituto per le Applicazioni

del Calcolo "Mauro Picone"

Sezione di Napoli

Consiglio Nazionale delle Ricerche

via Pietro Castellino 111

80131 Napoli, Italy
Department of Mathematics Shivaji College University of Delhi Raja Garden, Delhi 110027, India E-mail: babita.gupta@hotmail.com

Department of Mathematics Deshbandhu College University of Delhi

Kalkaji, New Delhi 110019, India E-mail: pankajkrjain@hotmail.com

Received June 22, 2007

Revised version April 18, 2008 